\title{
Risk factors of suprasternal lymph node metastasis in papillary thyroid carcinoma with clinical lateral cervical lymph node metastasis
}

\author{
Hyun-Keun Kwon $^{1} \wedge$, Yong-Il Cheon ${ }^{1} \wedge$, Sung-Chan Shin ${ }^{1} \wedge$, Eui-Suk Sung ${ }^{2} \wedge$, Jin-Choon Lee ${ }^{2}$, \\ In Ju Kim ${ }^{3}$, Byung-Joo Lee ${ }^{1 \wedge}$
}

${ }^{1}$ Department of Otorhinolaryngology-Head and Neck Surgery, College of Medicine, Pusan National University and Biomedical Research Institute, Pusan National University Hospital, Busan, Korea; ${ }^{2}$ Department of Otorhinolaryngology-Head and Neck Surgery, College of Research Institute for Convergence of Biomedical Science and Technology, Pusan National University Yangsan Hospital, Yangsan, Korea; ${ }^{3}$ Department of Internal medicine, College of Medicine and Medical Research Institute, Pusan National University Hospital, Busan, Korea

Contributions: (I) Conception and design: HK Kwon, IJ Kim, BJ Lee; (II) Administrative support: JC Lee, BJ Lee; (III) Provision of study materials or patients: HK Kwon, BJ Lee; (IV) Collection and assembly of data: YI Cheon, SC Shin, ES Sung; (V) Data analysis and interpretation: HK Kwon, JC Lee, IJ Kim; (VI) Manuscript writing: All authors; (VII) Final approval of manuscript: All authors.

Correspondence to: Byung-Joo Lee. Department of Otorhinolaryngology-Head and Neck Surgery, College of Medicine, Pusan National University and Biomedical Research Institute, Pusan National University Hospital, 179 Gudeok-ro Seo-gu Busan 49241, Korea. Email: voiceleebj@gmail.com.

Background Metastatic lymph nodes are occasionally found in suprasternal lymph nodes in patients with papillary thyroid cancer (PTC). However, limited studies have examined these lymph nodes thus far. Therefore, we investigated the frequency and risk factors of suprasternal lymph node metastasis in patients with PTC and lateral cervical lymph node metastasis.

Methods: A total of 85 patients with cN1b PTC underwent total thyroidectomy, central neck dissection, and ipsilateral selective neck dissection including suprasternal lymph node dissection. We analyzed the correlation between suprasternal lymph node metastasis and sex, age, tumor characteristics, and cervical lymph node metastasis status.

Results: Eleven (12.9\%) patients had pathological suprasternal lymph node metastasis. Suprasternal lymph node metastasis was associated with tumors located in the inferior pole of the thyroid gland and level IV lymph node metastasis ( $\mathrm{P}=0.005$ and 0.014 , respectively). Receiver operating characteristic curve analysis indicated that two or more level IV metastatic lymph nodes had the best predictive value for suprasternal lymph node metastasis $(\mathrm{P}<0.001)$.

Conclusions: In patients with cN1b PTC, especially those with tumors in the inferior pole of the thyroid gland or level IV lymph node metastasis, greater attention should be paid to the suprasternal lymph nodes and suprasternal lymph node dissection should be routinely included as part of selective neck dissection.

Keywords: Papillary thyroid carcinoma (PTC); suprasternal lymph node; cervical lymph node metastasis; neck dissection; risk factor

Submitted Mar 18, 2020. Accepted for publication Nov 07, 2020.

doi: 10.21037 /gs-20-368

View this article at: http://dx.doi.org/10.21037/gs-20-368

\footnotetext{
^ ORCID: Hyun-Keun Kwon, 0000-0003-4089-8639; Yong-Il Cheon, 0000-0003-0288-0542; Sung-Chan Shin, 0000-0003-2329-0648;

Eui-Suk Sung, 0000-0001-8752-3426; Jin-Choon Lee, 0000-0002-5629-4277; Byung-Joo Lee, 0000-0001-7091-6688.
} 


\section{Introduction}

Thyroid cancer is a common endocrine tumor. Papillary thyroid carcinoma (PTC) is the most common malignancy that occurs in thyroid follicular cells. The majority of PTC patients have a good prognosis with low mortality, but cervical lymph node metastases are common $(1,2)$. Most cervical lymph node metastases of PTC occur sequentially. The tumor cell spreads from the thyroid gland to the central and lateral lymph nodes on the ipsilateral side of the tumor, followed by metastasis to the opposite cervical lymph $\mathrm{n}$ ode (3). However, PTC located in the upper pole of the thyroid gland may present with lateral lymph node metastasis without central lymph node metastasis, which is often referred to as "skip metastasis" (4). According to previous studies, about $20-90 \%$ of PTC patients have cervical lymph node metastasis. The impact of cervical lymph node metastasis on overall survival is still controversial; however, it is known to increase the risk of locoregional recurrence (5-10). Therefore, complete resection of metastatic cervical lymph nodes is important to reduce locoregional recurrence of PTC.

The central neck compartment is bounded superiorly by the hyoid bone, laterally by the carotid arteries, anteriorly by the superficial layer of the deep cervical fascia, and posteriorly by the deep layer of the deep cervical fascia (11), not including the nodes anterior to the strap musculature. The anatomic boundary of the medial border of level III and IV lymph nodes is the lateral border of the sternohyoid muscle (12). The space anterior to the sternohyoid muscle, posterior to the sternocleidomastoid muscle, medial to the lateral border of the sternohyoid muscle, and superior to the clavicle and sternum does not fall under the normal subdivisions of the central and lateral neck compartment (Figure 1). This space is called the suprasternal space, and suprasternal lymph node metastases are occasionally observed on preoperative ultrasonography (US) or computed tomography (CT) in patients with recurrent PTC (Figure 2).

Thus far, limited studies have investigated the clinical significance of suprasternal lymph nodes. Therefore, we investigated the frequency and risk factors of suprasternal lymph node metastasis in PTC patients with lateral cervical lymph node metastasis.

We present the following article in accordance with the STROBE reporting checklist (available at http://dx.doi. org/10.21037/gs-20-368).

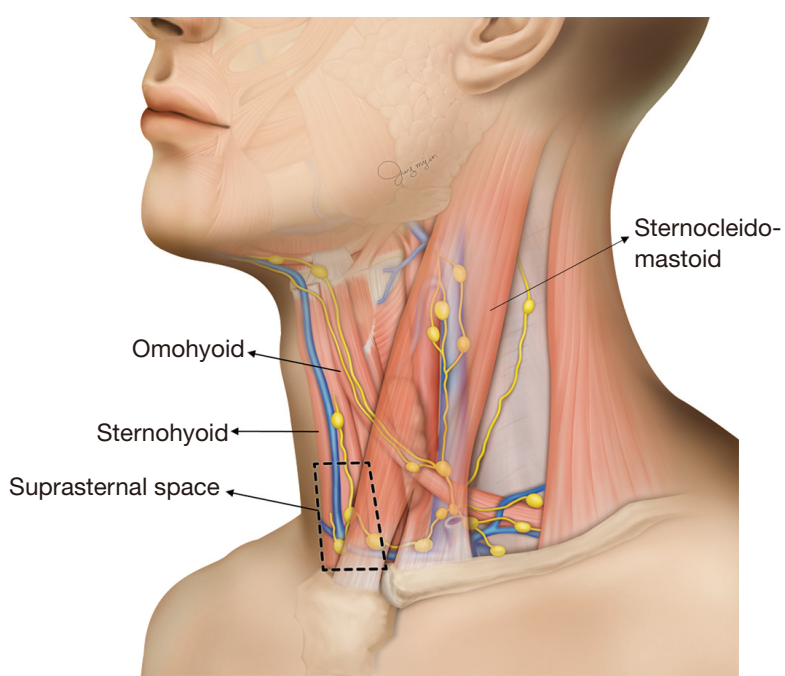

Figure 1 Schematic illustration of the suprasternal space. The suprasternal space is anterior to the sternohyoid muscle, posterior to the sternocleidomastoid muscle, medial to the lateral border of the sternohyoid muscle, and superior to the clavicle and sternum which does not fall under the normal subdivisions of the central and lateral neck compartment.

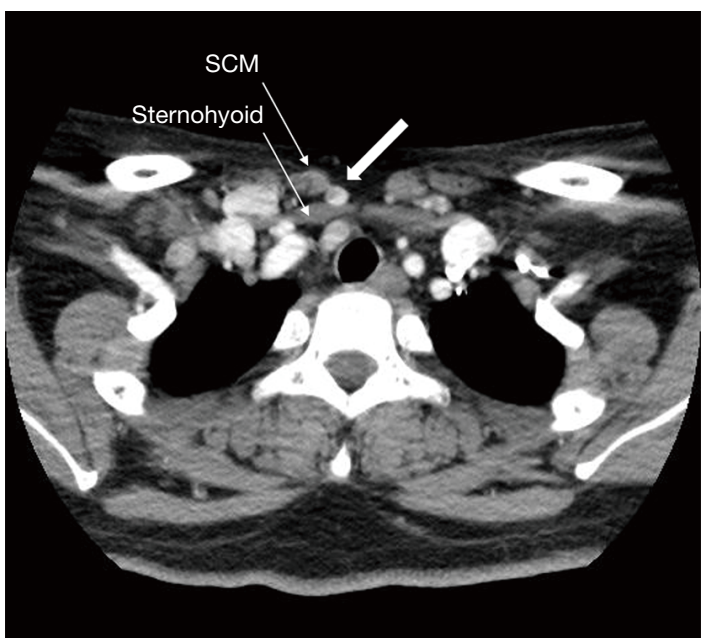

Figure 2 Preoperative computed tomography of suprasternal lymph node metastasis. Enhanced lymph node (white arrow) can be found between the sternocleidomastoid and sternohyoid muscles.

\section{Methods}

\section{Patients}

Data on a total of 85 patients with cN1b PTC [29 men and 
56 women, age: 17-83 (mean, 49.51) years] who underwent total thyroidectomy, central neck dissection, and ipsilateral selective neck dissection including suprasternal lymph node dissection at the Pusan National University Hospital from January 1, 2012 to January 31, 2018 were reviewed. The inclusion criteria were: (I) PTC with lateral cervical lymph node metastasis; (II) no previous treatment with radioactive iodine, radiotherapy, or chemotherapy prior to surgery; and (III) underwent suprasternal lymph node dissection. Patients who had undergone previous thyroidectomy or revision neck dissection were excluded. The study was conducted in accordance with the Declaration of Helsinki (as revised in 2013). This study was approved by the Institutional Review Board (IRB No. H-2002-003-087) and individual consent for this retrospective analysis was waived.

\section{Association of clinicopathological factors with suprasternal lymph node metastasis}

Associations between suprasternal lymph node metastasis and clinicopathological factors, including age, sex, tumor size, primary tumor location, extrathyroidal extension (ETE), multifocality, vascular invasion, lateral cervical lymph node metastasis, central compartment lymph node metastasis was investigated. The site of primary tumor was categorized as either the inferior or noninferior pole of the thyroid gland based on the preoperative US, CT, and postoperative pathological results. ETE was observed during surgery and confirmed with postoperative pathological results. Lymph node ratio (LNR) was also investigated. LNR was defined as the number of positive lymph nodes divided by the total number of lymph nodes harvested.

\section{Statistical analysis}

Chi-square test and Fisher's exact test were used as appropriate to identify risk factors for suprasternal lymph node metastasis. Receiver operating characteristic (ROC) curve analysis was performed to determine the predictability of suprasternal lymph node metastasis. The area under the ROC curve (AUC) with 95\% confidence interval (CI) was reported. $\mathrm{P}<0.05$ was considered statistically significant. SPSS version 21 (SPSS Inc., Chicago, IL) software was used for all analyses.

\section{Results}

All patients underwent successful surgical treatment, and no recurrence was observed during the follow-up period. The mean tumor size was $14.9 \pm 8.9 \mathrm{~mm}$. The most common pathological $\mathrm{T}$ stage was T3 $(83.5 \%)$, followed by $\mathrm{T} 1$ $(15.3 \%)$. The rate of suprasternal lymph node metastasis was $12.9 \%(11 / 85)$, and the mean number of dissected suprasternal lymph nodes was 1.4 (range, $0-4)$. More than one suprasternal lymph node was dissected in $76.5 \%(65 / 85)$ of patients.

Correlations between suprasternal lymph node metastasis and clinicopathologic factors were analyzed. Tumor location at the inferior pole was significantly associated with suprasternal lymph node metastasis $(\mathrm{P}=0.005)$. All patients with suprasternal lymph node metastasis had ETE, but the relationship was not statistically significant. Additionally, no significant association was found between suprasternal lymph node metastasis and age, sex, tumor size, multifocality, or vascular invasion (Table 1).

All patients had lateral lymph node metastasis, and $18.8 \%(16 / 85)$ of patients had lateral lymph node metastasis without central lymph node metastasis. These patients had no suprasternal lymph node metastasis. We analyzed the relationship between cervical lymph node metastasis and suprasternal lymph node metastasis. All patients with suprasternal lymph node metastasis had level IV and level VI nodal metastasis. However, only level IV nodal metastasis was significantly associated with suprasternal lymph node metastasis $(\mathrm{P}=0.014)$. No significant association was found between suprasternal lymph node metastasis and level II, level III, or level VI nodal metastasis (Table 2).

When examining the number of harvested and positive lymph nodes in patients with and without suprasternal lymph node metastasis, only the number of positive level IV lymph nodes was significantly different between the two groups $(\mathrm{P}<0.001)$. LNR of level IV and VI was significantly different between the two groups $(\mathrm{P}=0.002,0.009)$ (Table 3). To determine the optimal cut-off for the number of positive level IV lymph nodes, a ROC curve was constructed. ROC curve analysis indicated that $\geq 2$ positive lymph nodes had the best predictive value for suprasternal lymph node metastasis, with an AUC of 0.853 (95\% CI: 0.760-0.921, $\mathrm{P}<0.001$ ) (Figure 3). Also, the optimal cut-off for the LNR of level IV and VI lymph node was analyzed. ROC curve analysis indicated that the optimal cut-off value for LNR of level IV and VI was 0.125 and 0.357, with an AUC of 0.830 (95\% CI: $0.734-0.903, \mathrm{P}<0.001)$ and 0.741 (95\% CI: $0.634-0.830, \mathrm{P}<0.001$ ) (Figure 3).

In all patients, suprasternal lymph node dissection took approximately 5 minutes, and there were no specific 
Table 1 Factors related to suprasternal lymph node metastasis

\begin{tabular}{|c|c|c|c|}
\hline Parameter & \multicolumn{2}{|c|}{ Suprasternal lymph node } & $P$ value \\
\hline Sex & & & 1.000 \\
\hline Male & 4 & 25 & \\
\hline Female & 7 & 49 & \\
\hline$<55$ & 6 & 41 & \\
\hline$\geq 55$ & 5 & 33 & \\
\hline Primary tumor size, $\mathrm{cm}$ & & & 0.275 \\
\hline$<1$ & 1 & 22 & \\
\hline Noninferior pole & 4 & 59 & \\
\hline Inferior pole & 7 & 15 & \\
\hline Extrathyroidal extension & & & 0.198 \\
\hline Yes & 11 & 60 & \\
\hline No & 0 & 14 & \\
\hline Multifocality & & & 0.174 \\
\hline Yes & 6 & 23 & \\
\hline No & 5 & 51 & \\
\hline
\end{tabular}

*, statistically significant $(\mathrm{P}<0.05)$.

intraoperative or postoperative complications such as bleeding or pain.

\section{Discussion}

The suprasternal space, which is also known as the "Burns space," is a narrow space between the superficial and deep layers of the investing layers of the deep cervical fascia superior to the manubrium of the sternum (13). According to Gray's Anatomy, it contains a small amount of areolar tissue, the lower parts of the anterior jugular veins, and the jugular venous arch, as well as the sternal heads of the sternocleidomastoid muscles and occasionally lymph nodes (14). Because of the anatomical location of the suprasternal space, invasion to the clavicle or sternum is thought to be related. However, in our experience, bone invasion in the clavicle or sternum was not observed in patients with PTC. The result of this study showed that suprasternal lymph node metastases are associated with tumors located in the inferior pole and level IV lymph node metastasis. The suprasternal lymph node metastasis can be predicted when there are two or more level IV lymph node metastasis. Level IV LNR $>0.125$ and level VI LNR $>0.357$ were also associated with suprasternal lymph node metastasis.

Sun et al. first defined the lymph node area between the sternocleidomastoid and sternohyoid muscle as follows: its anterior boundary as the sternocleidomastoid muscle, its posterior as the sternohyoid muscle, its superior as the intersection of sternocleidomastoid and sternohyoid muscle, 
Table 2 Relationship between cervical lymph node metastasis and suprasternal lymph node metastasis

\begin{tabular}{|c|c|c|c|}
\hline Parameter & \multicolumn{2}{|c|}{ Suprasternal lymph node } & $P$ value \\
\hline Level II metastasis & & & 0.103 \\
\hline Yes & 9 & 39 & \\
\hline No & 2 & 35 & \\
\hline Yes & 10 & 56 & \\
\hline No & 1 & 18 & \\
\hline Level IV metastasis & & & $0.014^{*}$ \\
\hline Yes & 11 & 47 & \\
\hline Yes & 11 & 58 & \\
\hline No & 0 & 16 & \\
\hline
\end{tabular}

*, statistically significant $(\mathrm{P}<0.05)$.

Table 3 Relationship between cervical lymph node ratio (LNR) and suprasternal lymph node metastasis

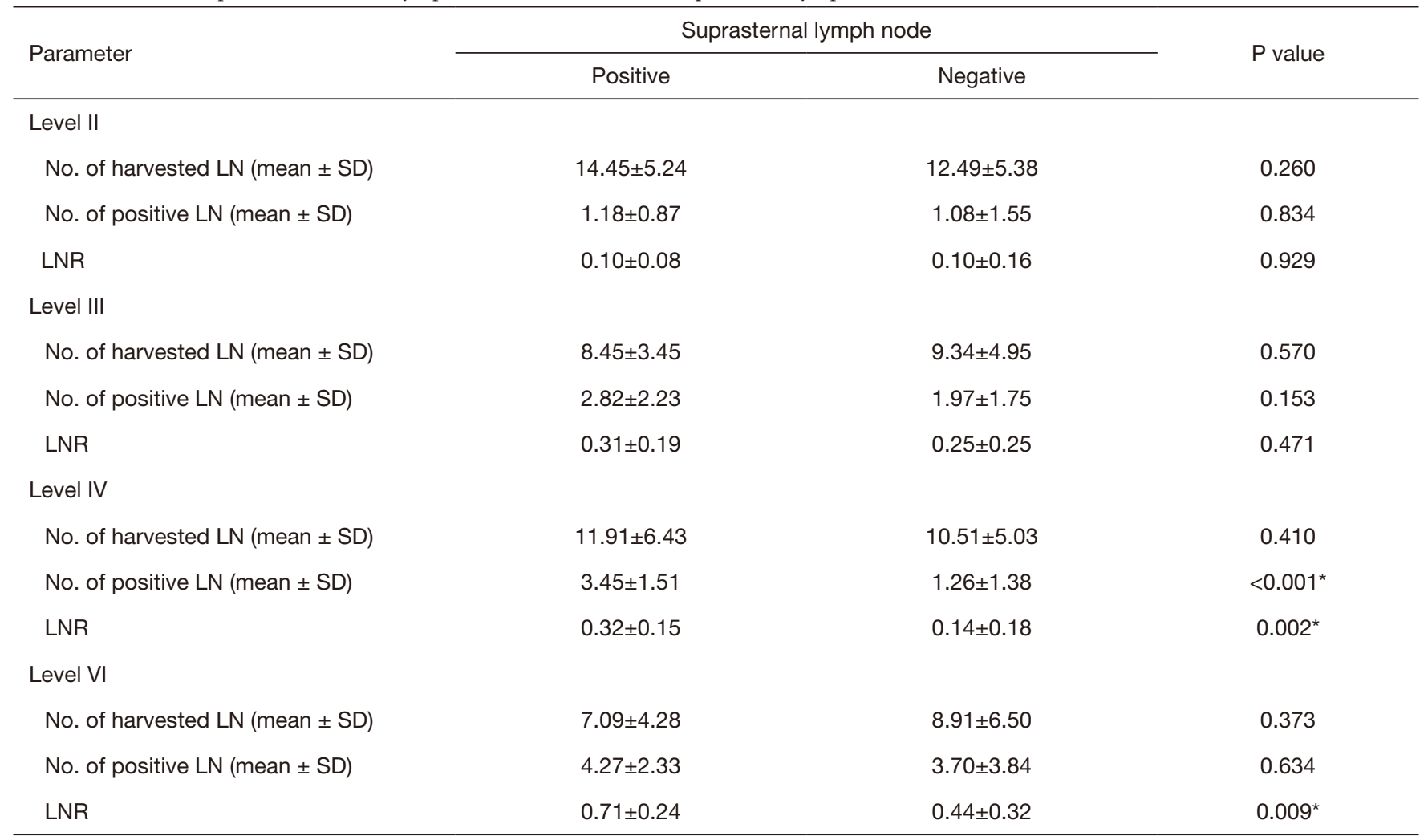

${ }^{*}$, statistically significant $(\mathrm{P}<0.05)$. 

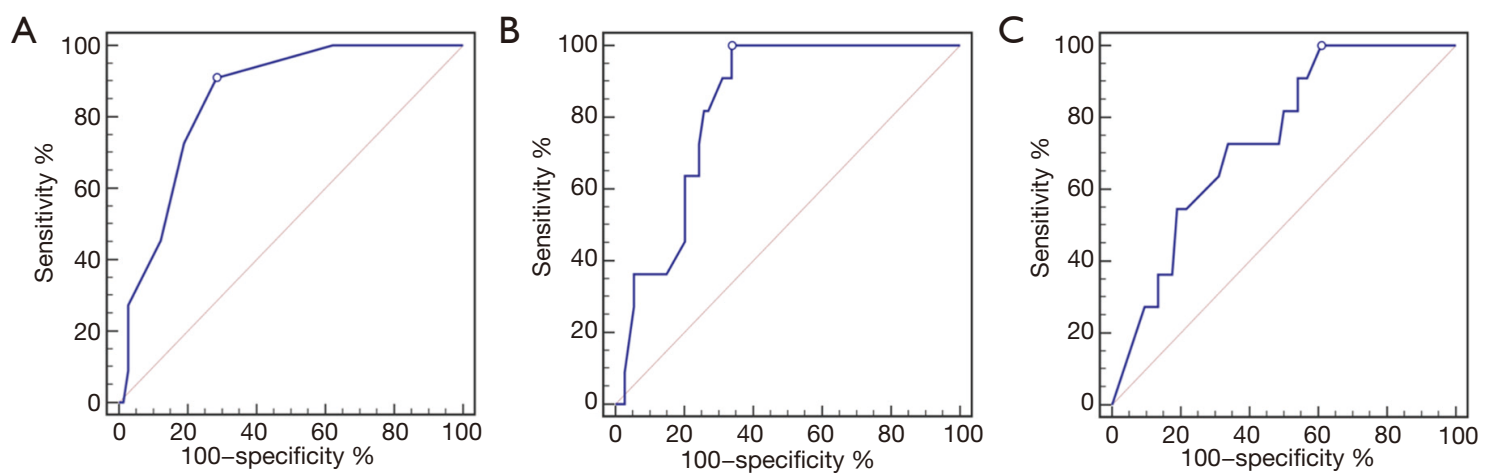

Figure 3 Receiver operating characteristic (ROC) curves for the association between cervical lymph node and suprasternal lymph node metastasis. (A) Two or more metastases in the level IV lymph node had the best predictive value for suprasternal lymph node metastasis, with an area under the ROC curve AUC of 0.853 [95\% confidence interval (CI) $0.760-0.921, \mathrm{P}<0.001$ ]; (B) the level IV lymph node ratio $(\mathrm{LNR})>0.125$ had a predictive value for suprasternal lymph node metastasis, with an AUC of 0.830 (95\% CI: 0.734-0.903, P<0.001); (C) The level VI LNR $>0.357$ had a predictive value for suprasternal lymph node metastasis, with an AUC of 0.741 (95\% CI: 0.634-0.830, P<0.001).

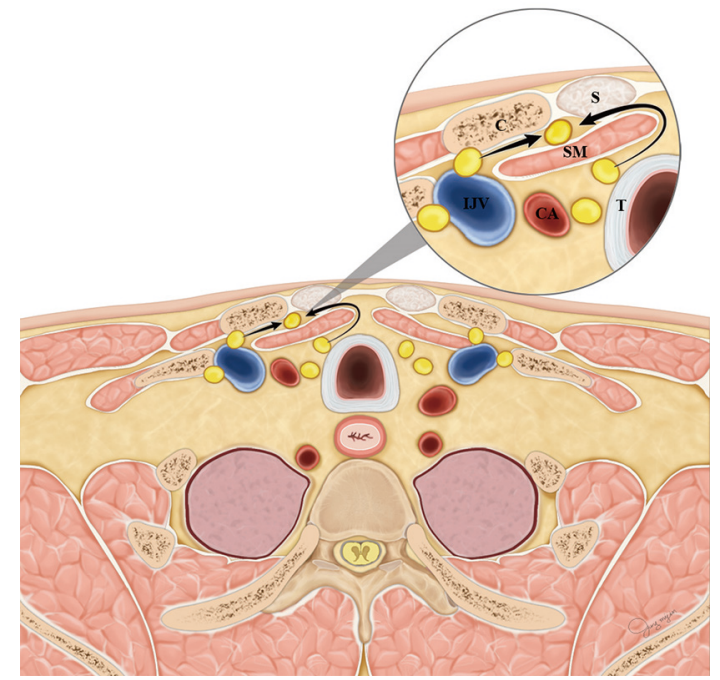

Figure 4 Schematic illustration of two pathways for suprasternal lymph node metastasis. Lymph nodes from level IV would move medially (straight arrow), while those moving from level VI would move laterally (curved arrow). S, sternum; C, clavicle; T, trachea; SM, strap muscle.

and its inferiors as suprasternal fossa and clavicle, and its medial and lateral boundaries formed by the medial and lateral borders of the sternohyoid muscle, respectively (13). They reported that this space is part of the suprasternal space and found up to 5 (average, 3) lymph nodes on pathological examination. In this study, up to 4 (average, 1.4) lymph nodes were dissected and confirmed in the suprasternal space. Although the number of lymph nodes is small, it is certain that lymph nodes are present in the suprasternal space.

The importance of lymph node metastases in prognosis have been reported in low-risk as well as high-risk patients $(15-17)$. Selective neck dissection should be performed on patients who have PTC with clinically apparent cervical lymph node metastasis detected on palpation or imaging studies (18-20). The extent of selective neck dissection is still controversial, but a thorough lymph node dissection is required. Therefore, suprasternal lymph node metastasis is rare but should be routinely included in the selective neck dissection because there are few complications during dissection.

The etiology of suprasternal lymph node metastasis is not clear. Sun et al. reported that, without exception, patients with suprasternal space metastasis also had lateral cervical lymph node metastasis, and level III and IV metastases were significantly correlated with suprasternal space metastasis (13). They speculated that suprasternal space metastasis could be a result of the increasing tumor load after lateral cervical metastasis (13). Homma et al. speculate that fibrofatty tissue, including the level III and IV metastatic lymph nodes, moves into the suprasternal space gradually due to the daily motion of the neck (21). We present two theories through the anterior jugular node chain. First, level IV metastatic lymph nodes move into the suprasternal space laterally. Second, central lymph nodes, especially the pretracheal and paratracheal metastatic lymph nodes, move into the suprasternal space medially (Figure 4). In this study, all patients with suprasternal lymph 
node metastasis had level IV and VI metastasis. However, only level IV metastasis was significantly correlated with suprasternal lymph node metastasis, and two or more level IV metastatic lymph nodes had the best predictive value. Therefore, it is important to dissect the suprasternal lymph node adjacent to the level IV lymph node, as the first theory is more convincing.

Suprasternal lymph node metastasis can be recognized by preoperative ultrasonography or CT; however, there is little clinical interest. Therefore, suprasternal lymph nodes are not routinely dissected because they are not included in the central and lateral neck compartment. Sun et al. reported that the positive rate for the suprasternal space was $22.6 \%$ among 115 patients with clinically nodepositive PTC who underwent neck dissection that included the suprasternal space. In their study, a primary site in the inferior pole of the thyroid gland, lateral nodal metastasis, and level III and level IV nodal metastasis were correlated with suprasternal lymph node metastasis (13). In a study by $\mathrm{Yu} e t \mathrm{al}$., the metastasis rate of the suprasternal space was $20.7 \%$ among 140 patients with pathologically nodepositive PTC. In their study, a primary site in the inferior portion of the thyroid gland, strap muscle invasion, level IV metastasis, and lymph node metastasis between the sternocleidomastoid and sternohyoid muscles were independent risk factors for suprasternal lymph node metastasis (22). In our study, the positive rate for the suprasternal space was $12.9 \%$ among 85 patients, and a primary site in the inferior pole of the thyroid gland and level IV metastasis were correlated with suprasternal lymph node metastasis.

The number of metastatic lymph nodes and LNR are risk factors for locoregional recurrence in PTC $(9,10)$. Suprasternal lymph node metastasis was associated with level IV lymph node metastasis and can be predicted when there are two or more level IV lymph node metastasis. Level IV LNR $>0.125$ and level VI LNR $>0.357$ were also associated with suprasternal lymph node metastasis. Therefore, a large number of level VI metastases as well as level IV metastases is a highly correlated with suprasternal lymph node metastasis. In this study, it did not take much longer to dissect the suprasternal lymph nodes, and there were no intraoperative or postoperative complications such as bleeding or pain.

PTC has an excellent prognosis, with a 10 -year overall survival rate $>90 \%$ (22). However, in rare cases, nodal recurrence may occur in the suprasternal lymph nodes. Because locoregional recurrence can affect quality of life (23), it is important to reduce the risk of locoregional recurrence by complete resection of the lymph nodes during surgery (24). Although the frequency of suprasternal lymph node metastasis is low, suprasternal lymph node dissection is less invasive, easier to achieve, and less time consuming than central compartment dissection, which can increase the risk of recurrent laryngeal nerve palsy and hypocalcemia (25). To reduce locoregional recurrence in patients with PTC and positive lateral nodes, we suggest performing a suprasternal lymph node dissection while performing selective neck dissection.

The main limitation of this study is that it involved a small number of patients from a single-institution; hence, the findings may have been underestimated or overestimated. In addition, this study may have some inevitable bias owing to its retrospective nature. Therefore, a prospective study with a larger cohort is needed to further examine the clinical significance of suprasternal lymph node dissection in patients with PTC.

\section{Conclusions}

In patients with $\mathrm{cN} 1 \mathrm{~b} \mathrm{PTC}$, especially those with tumors in the inferior pole of the thyroid gland or level IV lymph node metastasis, suprasternal lymph node dissection should be routinely included as part of selective neck dissection.

\section{Acknowledgments}

We would like to thank Editage (www.editage.co.kr) for English language editing.

Funding: This work was funded by the National Research Foundation of Korea (NRF) grant funded by the Korean government (MSIT) (NRF- 2017R1E1A1A01074316).

\section{Footnote}

Reporting Checklist: The authors have completed the STROBE reporting checklist. Available at http://dx. doi. org/10. 21037/gs-20-368

Data Sharing Statement: Available at http://dx.doi. org/10.21037/gs-20-368

Conflicts of Interest: All authors have completed the ICMJE uniform disclosure form (available at http://dx.doi. org/10.21037/gs-20-368). The authors have no conflicts of interest to declare. 
Ethical Statement: The authors are accountable for all aspects of the work in ensuring that questions related to the accuracy or integrity of any part of the work are appropriately investigated and resolved. The study was conducted in accordance with the Declaration of Helsinki (as revised in 2013). The Institutional Review Board of Pusan National University Hospital approved this study (No. $\mathrm{H}-2002-003-087)$ and waived the requirement for informed consent.

Open Access Statement: This is an Open Access article distributed in accordance with the Creative Commons Attribution-NonCommercial-NoDerivs 4.0 International License (CC BY-NC-ND 4.0), which permits the noncommercial replication and distribution of the article with the strict proviso that no changes or edits are made and the original work is properly cited (including links to both the formal publication through the relevant DOI and the license). See: https://creativecommons.org/licenses/by-nc-nd/4.0/.

\section{References}

1. Ito Y, Kudo T, Kobayashi K, et al. Prognostic factors for recurrence of papillary thyroid carcinoma in the lymph nodes, lung, and bone: analysis of 5,768 patients with average 10-year follow-up. World J Surg 2012;36:1274-8.

2. Moo TA, McGill J, Allendorf J, et al. Impact of prophylactic central neck lymph node dissection on early recurrence in papillary thyroid carcinoma. World J Surg 2010;34:1187-91.

3. Machens A, Hinze R, Thomusch O, et al. Pattern of nodal metastasis for primary and reoperative thyroid cancer. World J Surg 2002;26:22-8.

4. Lee YS, Shin SC, Lim YS, et al. Tumor locationdependent skip lateral cervical lymph node metastasis in papillary thyroid cancer. Head Neck 2014;36:887-91.

5. Cooper DS, Doherty GM, Haugen BR, et al. Revised American Thyroid Association management guidelines for patients with thyroid nodules and differentiated thyroid cancer. Thyroid 2009;19:1167-214.

6. Park YM, Wang SG, Shin DH, et al. Lymph node status of lateral neck compartment in patients with $\mathrm{N} 1 \mathrm{~b}$ papillary thyroid carcinoma. Acta Otolaryngol 2016;136:319-24.

7. Hay ID, Hutchinson ME, Gonzalez-Losada T, et al. Papillary thyroid microcarcinoma: a study of 900 cases observed in a 60-year period. Surgery 2008;144:980-7; discussion 987-8.

8. Podnos YD, Smith D, Wagman LD, et al. The implication of lymph node metastasis on survival in patients with welldifferentiated thyroid cancer. Am Surg 2005;71:731-4.

9. Choi SY, Cho JK, Moon JH, et al. Metastatic Lymph Node Ratio of Central Neck Compartment Has Predictive Values for Locoregional Recurrence in Papillary Thyroid Microcarcinoma. Clin Exp Otorhinolaryngol 2016;9:75-9.

10. Zheng CM, Ji YB, Song CM, et al. Number of Metastatic Lymph Nodes and Ratio of Metastatic Lymph Nodes to Total Number of Retrieved Lymph Nodes Are Risk Factors for Recurrence in Patients With Clinically Node Negative Papillary Thyroid Carcinoma. Clin Exp Otorhinolaryngol 2018;11:58-64.

11. Carty SE, Cooper DS, Doherty GM, et al. Consensus statement on the terminology and classification of central neck dissection for thyroid cancer. Thyroid 2009;19:1153-8.

12. Robbins KT, Clayman G, Levine PA, et al. Neck dissection classification update: revisions proposed by the American Head and Neck Society and the American Academy of Otolaryngology-Head and Neck Surgery. Arch Otolaryngol Head Neck Surg 2002;128:751-8.

13. Sun G, Wang Y, Zhu Y, et al. Lymph node metastasis between sternocleidomastoid and sternohyoid muscle in clinically node-positive papillary thyroid carcinoma. Head Neck 2013;35:1168-70.

14. Standring S. Gray's anatomy: the anatomical basis of clinical practice, expert consult. 40th ed. UK: Churchill Livingstone; 2008.

15. Zaydfudim V, Feurer ID, Griffin MR, et al. The impact of lymph node involvement on survival in patients with papillary and follicular thyroid carcinoma. Surgery 2008;144:1070-7; discussion 7-8.

16. Nixon IJ, Wang LY, Palmer FL, et al. The impact of nodal status on outcome in older patients with papillary thyroid cancer. Surgery 2014;156:137-46.

17. Adam MA, Pura J, Goffredo P, et al. Presence and Number of Lymph Node Metastases Are Associated With Compromised Survival for Patients Younger Than Age 45 Years With Papillary Thyroid Cancer. J Clin Oncol 2015;33:2370-5.

18. Noguchi S, Murakami N. The value of lymph-node dissection in patients with differentiated thyroid cancer. Surg Clin North Am 1987;67:251-61.

19. Shaha AR. Management of the neck in thyroid cancer. Otolaryngol Clin North Am 1998;31:823-31.

20. Bhattacharyya N. Surgical treatment of cervical nodal metastases in patients with papillary thyroid carcinoma. Arch Otolaryngol Head Neck Surg 2003;129:1101-4. 
21. Homma A, Hatakeyama H, Mizumachi T, et al. Lymph node metastasis in the suprasternal space from thyroid papillary cancer. International Cancer Conference Journal 2014;4:57-60.

22. Lundgren CI, Hall P, Ekbom A, et al. Incidence and survival of Swedish patients with differentiated thyroid cancer. Int J Cancer 2003;106:569-73.

23. Hughes CJ, Shaha AR, Shah JP, et al. Impact of lymph node metastasis in differentiated carcinoma of the thyroid: a matched-pair analysis. Head Neck 1996;18:127-32.

24. Noguchi S, Murakami N, Yamashita H, et al. Papillary thyroid carcinoma: modified radical neck dissection improves prognosis. Arch Surg 1998;133:276-80.

25. Chen L, Wu YH, Lee CH, et al. Prophylactic Central Neck Dissection for Papillary Thyroid Carcinoma with Clinically Uninvolved Central Neck Lymph Nodes: A Systematic Review and Meta-analysis. World J Surg 2018;42:2846-57.

Cite this article as: Kwon HK, Cheon YI, Shin SC, Sung ES, Lee JC, Kim IJ, Lee BJ. Risk factors of suprasternal lymph node metastasis in papillary thyroid carcinoma with clinical lateral cervical lymph node metastasis. Gland Surg 2021;10(2):512520. doi: $10.21037 /$ gs-20-368 